\title{
Phospholipid fatty acids of brain and liver are modified by $\alpha$-tocopherol and dietary fat in growing chicks
}

\author{
BY H. FUHRMANN AND H. P. SALLMANN \\ Institute of Physiological Chemistry, Hannover Veterinary School, Buenteweg 17, \\ D-30559 Hannover, Germany
}

(Received 6 July 1995-Revised 16 October 1995-Accepted 15 November 1995)

\begin{abstract}
Dietary fatty acids modify phospholipid fatty acids in brain and liver of growing chickens post-hatching. The effect of vitamin $E$ deficiency on this process is unknown and may be relevant to the pathogenesis of chick nutritional encephalomalacia (NE). Therefore laying hens received a diet low in vitamin $\mathbf{E}$ (10 $\mathrm{mg} \alpha$-tocopherol $/ \mathrm{kg}$ feed). Resulting chicks were assigned to nine dietary groups each fed with either oleic $(18: 1 n-9,58 \mathrm{~g} / \mathrm{kg})$, linoleic $(18: 2 n-6,57 \mathrm{~g} / \mathrm{kg})$ or linolenic $(18: 3 n-3,56 \mathrm{~g} / \mathrm{kg})$ acid together with 5,25 or $125 \mathrm{mg} \alpha$-tocopherol/kg feed. $\mathrm{NE}$ affecting the cerebellum only occurred in the group given linoleic acid and $5 \mathrm{mg} \alpha$-tocopherol $/ \mathrm{kg}$. In 1-d-old chicks and after 1 and 2 weeks the phospholipid fatty acid composition of liver, cerebrum and cerebellum (additionally after 3 weeks) was determined. The feed fatty acids were incorporated into the liver very efficiently during the first week of life. Unsaturation of liver membranes decreased in the order dietary linolenic $>$ linoleic $>$ oleic acid. In liver, also, the effect of $\alpha$-tocopherol supplementation on phospholipid fatty acids was most pronounced. The unsaturation index increased during deficiency, whereas $n-9$ fatty acids decreased. In the chicken brain the alterations were delayed and less distinct. The cerebellum phospholipids were rich in $n-9$ fatty acids and as a whole more saturated in comparison with the cerebrum. Cerebellar unsaturation increased when linolenic or linoleic acid was given. However, NE-producing dietary conditions were not accompanied by specific alterations in cerebellar phospholipid fatty acids due to the $\alpha$-tocopherol content of the diet. Rather the alterations of membrane fatty acids in the liver seem to play a role in the pathogenesis of $\mathrm{NE}$.
\end{abstract}

Lipid peroxidation: Encephalomalacia: Vitamin $\mathbf{E}$

A high concentration of polyunsaturated fatty acids in membranes causes susceptibility to peroxidative degradation. This is mediated by free radicals generated during normal cellular events or during metabolism of xenobiotics (Aust et al. 1993). Susceptibility to lipid peroxidation is also greatly influenced by tissue concentration of $\alpha$-tocopherol, which is the most important, if not the only, radical scavenger within membranes in vivo (DiMascio et al. 1991).

One of the classical vitamin E-deficiency syndromes in the chick is nutritional encephalomalacia (NE). It is readily produced by vitamin E-deficient diets containing polyunsaturated fatty acids of the $n-6$ series (Budowski et al. 1987). From the chick (Fuhrmann \& Sallmann, 1995) and other species (Bourre \& Clement, 1991) it is known that the brain is a poor responder to dietary $\alpha$-tocopherol. In broiler chickens NE affects only the cerebellum (Hassan et al. 1990), leading to ataxia, prostration and death. The disease normally occurs during the second or third week after hatching. It has been proposed that this is related to the cerebel!ar changes in polyunsaturated fatty acids during the third week of life (Budowski et al. 1987). Therefore, the present study was planned to investigate whether $\alpha$-tocopherol modifies the incorporation of dietary fat into tissue phospholipid fatty acids in such a way as to clarify the role of dietary $n-6$ fatty acids in the pathogenesis 
of NE. As an approach to the problem we used 1-d-old broiler chicks obtained from hens with a marginal supply of $\alpha$-tocopherol. These chicks were reared on diets differing in $\alpha$ tocopherol content and fat source. A time course was implemented during 3 weeks posthatching to study in detail the fatty acid pattern in cerebellum in comparison with that in cerebrum and liver.

\section{MATERIALS AND METHODS}

To obtain 1-d-old chicks regularly low in tissue $\alpha$-tocopherol, a breeder flock (Lohmann Meat; Lohmann, Cuxhaven, Germany) received a vitamin E-poor diet $(10 \mathrm{mg} \alpha-$ tocopherol $/ \mathrm{kg}$ ) based on barley, wheat starch, cassava and soyabean meal, starting from the 13 th week of life. The diet contained $50 \mathrm{~g}$ fat $/ \mathrm{kg}$ with $(\mathrm{g} / 100 \mathrm{~g}$ total fatty acids) saturated 33, monounsaturated 34, 18:2n-6 19 and $n-3$ fatty acids 11 . From the 30 th week the eggs were collected for 8 (SD 2) $d$ and incubated. The newly hatched chicks received the basal semi-purified diet (Table 1) without fat and $\alpha$-tocopherol for $48 \mathrm{~h}$. Thereafter the chicks were randomly divided into groups of 26-29 animals, and each group was randomly allocated to one of the nine dietary treatments. From that time on the animals received the experimental diets containing $50 \mathrm{~g}$ fat $/ \mathrm{kg}$ and the $\alpha$-tocopherol supplement (Table 1 ). The compositions of the different dietary fats and the resulting fatty acid patterns are listed in Table 2. The fatty acid compositions of the single fat components are given in Table 3 . The tocopherol content of the basal diet and the fat components were measured. The fat mixtures were adjusted to $100 \mathrm{mg} \alpha$-tocopherol $/ \mathrm{kg}$ fat with $\mathrm{RRR}$ - $\alpha$-tocopherol. The vitamin $\mathrm{E}$ premixes contained $0,0.5$ and $2.5 \mathrm{mg} \alpha$-tocopherol as all-rac- $\alpha$-tocopheryl acetate $/ \mathrm{g}$ maize starch to give 5,25 and $125 \mathrm{mg} \alpha$-tocopherol $/ \mathrm{kg}$ in the final feed. The feed (basal diet + supplements) was remixed every week and stored in the dark at $-22^{\circ}$.

Each dietary group was housed in a separate wire cage. The environment in the fully airconditioned room was kept constant at $32 \pm 1^{\circ}$ during the first week followed by a weekly reduction by $2-3^{\circ}$ down to $25^{\circ}$. Humidity was maintained at $60 \pm 5 \%$; light was provided for $24 \mathrm{~h}$. After 1, 2 and 3 weeks one third of the animals were randomly selected for examination. The birds were fasted for $16 \mathrm{~h}$ before they were anaesthetized by intramuscular injection of Metomidat- $\mathrm{HCl}$ (Hypnodil ${ }^{\circledR}$; Janssen, Duesseldorf, Germany: $7.5 \mathrm{mg} / \mathrm{kg}$ body weight) killed and completely bled. Liver, cerebrum and cerebellum were homogenized in ice-cold $50 \mathrm{mM}-\mathrm{KCl}(\mathrm{pH} \mathrm{7.4)}$ including butylated hydroxytoluene (BHT) $(50 \mathrm{mg} / 1)$ for determination of phospholipid fatty acids and protein (Lowry et al. 1951). Samples were stored deep-frozen at $-80^{\circ}$ until further processing. Total lipids were extracted from the $100 \mathrm{~g} / 1$ homogenates using the Bligh \& Dyer (1959) method. A $0.8 \mathrm{ml}$ portion of the sample was quickly thawed and homogenized in $1 \mathrm{ml}$ chloroform containing $150 \mu \mathrm{g}$ phosphatidylcholine-C17 as the internal standard and $2 \mathrm{ml}$ methanol. The supernatant fraction from the centrifugation step $(1000 \mathrm{~g}$, at room temperature for $5 \mathrm{~min}$ ) was collected and mixed with $1 \mathrm{ml}$ chloroform and $1 \mathrm{ml}$ water. From the chloroform layer the phospholipids were separated by solid-phase extraction on $500 \mathrm{mg}$ aminopropyl columns (Kaluzny et al. 1985). The columns were preconditioned with $4 \mathrm{ml}$ hexane, then $0.5 \mathrm{ml}$ of the extract was applied. Neutral lipids were eluted with $4 \mathrm{ml}$ chloroformisopropanol $(98: 2, \mathrm{v} / \mathrm{v})$ and free fatty acids with $4 \mathrm{ml}$ diethyl ether-glacial acid $(96: 4, \mathrm{v} / \mathrm{v})$. Phosphatidylcholine, phosphatidylethanolamine and sphingomyelin were eluted with $4 \mathrm{ml}$ methanol, phosphatidylserine and phosphatidylinositol with methanol-ammonia $(250 \mathrm{ml} / \mathrm{l})(95: 5, \mathrm{v} / \mathrm{v})$. The combined extracts were evaporated under a stream of $\mathrm{N}_{2}$ and dissolved again in $2 \mathrm{ml}$ chloroform-methanol $(25: 75, \mathrm{v} / \mathrm{v})$ containing $100 \mu \mathrm{g}$ BHT in tubes with Teflon-coated screw caps. For direct transesterification (Lepage \& Roy, 1986) $200 \mu 1$ acetylchloride was added slowly under continuous stirring. The tubes were heated for $1 \mathrm{~h}$ at $100^{\circ}$ with the caps closed. The reaction was stopped in an ice bath by adding, slowly, $4 \mathrm{ml}$ 
Table 1. Composition of the experimental diet $(\mathrm{g} / \mathrm{kg})$

\begin{tabular}{lr}
\hline \hline Basal diet & \\
Maize starch & 426 \\
Soyabean meal & 344 \\
Mineral mixture* & 70 \\
Cellulose & 40 \\
Amino acids and vitamins $\dagger$ & 20 \\
Supplements & 50 \\
Fat & 50 \\
Vitamin E premixture & \\
\hline
\end{tabular}

* Contained (g/kg feed): $\mathrm{CaHPO}_{4} \cdot 2 \mathrm{H}_{2} \mathrm{O} 19 \cdot 6, \mathrm{CaCO}_{3} 20 \cdot 5, \mathrm{KH}_{2} \mathrm{PO}_{4} 15 \cdot 2, \mathrm{NaHCO}_{3} 9 \cdot 6, \mathrm{MnSO}_{4} \cdot \mathrm{H}_{2} \mathrm{O} 0 \cdot 38$, $\mathrm{FeSO}_{4} .7 \mathrm{H}_{2} \mathrm{O} 0 \cdot 54, \mathrm{MgSO}_{4} 3 \cdot 28, \mathrm{KIO}_{3} 10 \cdot 0, \mathrm{CuSO}_{4} \cdot 5 \mathrm{H}_{2} \mathrm{O} 0 \cdot 036, \mathrm{ZnCO}_{3} 0 \cdot 16, \mathrm{CoCl}_{2} 0.0034, \mathrm{NaMoO}_{4} \cdot \mathrm{H}_{2} \mathrm{O} 0 \cdot 009$, $\mathrm{NiCl}_{2} \cdot \mathrm{H}_{2} \mathrm{O} 0 \cdot 13, \mathrm{Na}_{2} \mathrm{SeO}_{3} .5 \mathrm{H}_{2} \mathrm{O} 0.333 \mathrm{mg}$.

$\dagger$ Rovimix 428 (Hoffmann-La Roche, Basle, Switzerland) without vitamin E.

Table 2. Composition $(\mathrm{g} / \mathrm{kg}$ fat $)$ and fatty acid pattern $(\mathrm{g} / 100 \mathrm{~g}$ total fatty acids) of the fats used in the experimental diets

\begin{tabular}{|c|c|c|c|c|c|c|}
\hline \multirow[b]{2}{*}{ Diet } & \multirow[b]{2}{*}{ Fat source } & \multirow[b]{2}{*}{$\mathrm{g} / \mathrm{kg}$ fat } & \multicolumn{4}{|c|}{$\mathrm{g} / 100 \mathrm{~g}$ total fatty acids } \\
\hline & & & $n-3$ & $n-6$ & $n-9$ & SFA \\
\hline Linolenic acid-rich & $\begin{array}{l}\text { Linolenic acid } 62 \% * \\
\text { Tallow }\end{array}$ & $\begin{array}{l}860 \\
140\end{array}$ & $\overline{56}$ & $\overline{14}$ & $\overline{18}$ & $\overline{11}$ \\
\hline Linoleic acid-rich & $\begin{array}{l}\text { Soyabean triacylglycerol } \dagger \\
\text { Safflower-seed oil } \ddagger \\
\text { Linolenic acid } 62 \%\end{array}$ & $\begin{array}{l}775 \\
125 \\
100\end{array}$ & $\overline{-}$ & $\overline{-}$ & $\frac{-}{16}$ & $\overline{-}$ \\
\hline Oleic acid-rich & $\begin{array}{l}\text { Lard§ } \\
\text { Oleic acid } 82 \% \\
\text { Linolenic acid } 62 \%\end{array}$ & $\begin{array}{r}670 \\
240 \\
90\end{array}$ & $\overline{-}$ & $\frac{-}{7}$ & $\overline{-}$ & $\overline{-}$ \\
\hline
\end{tabular}

SFA, saturated fatty acids.

* Serva, Heidelberg, Germany.

† Henkel, Düsseldorf, Germany.

‡ Lidl, Hannover, Germany.

$\S \mathrm{ICN}$, Meckenheim, Germany.

Merck, Darmstadt, Germany.

Table 3. Fatty acid composition $(\mathrm{g} / 100 \mathrm{~g}$ total fatty acids) of the fat components of the experimental diets*

\begin{tabular}{lcccrrr}
\hline $\begin{array}{c}\text { Fatty } \\
\text { acid }\end{array}$ & $\begin{array}{c}\text { Linolenic } \\
\text { acid }\end{array}$ & $\begin{array}{c}\text { Safflower-seed } \\
\text { oil }\end{array}$ & $\begin{array}{c}\text { Soyabean } \\
\text { oil }\end{array}$ & Lard & Tallow & \multicolumn{1}{c}{$\begin{array}{c}\text { Oleic } \\
\text { acid }\end{array}$} \\
\hline $14: 0$ & - & $4 \cdot 6$ & $0 \cdot 2$ & $1 \cdot 1$ & $3 \cdot 7$ & $2 \cdot 5$ \\
$16: 0$ & $4 \cdot 5$ & $5 \cdot 5$ & $9 \cdot 7$ & $25 \cdot 6$ & $31 \cdot 4$ & $4 \cdot 7$ \\
$16: 1 n-7$ & - & - & - & $3 \cdot 3$ & $1 \cdot 9$ & $0 \cdot 3$ \\
$18: 0$ & $3 \cdot 0$ & $2 \cdot 1$ & $4 \cdot 5$ & $10 \cdot 9$ & $24 \cdot 1$ & - \\
$18: 1 n-9$ & $16 \cdot 5$ & $9 \cdot 7$ & $22 \cdot 4$ & $50 \cdot 5$ & $35 \cdot 2$ & $82 \cdot 0$ \\
$18: 2 n-6$ & $15 \cdot 5$ & $75 \cdot 5$ & $55 \cdot 2$ & $7 \cdot 9$ & $1 \cdot 6$ & $4 \cdot 8$ \\
$18: 3 n-3$ & $59 \cdot 7$ & 0.9 & $3 \cdot 8$ & $0 \cdot 1$ & $0 \cdot 1$ & $0 \cdot 1$ \\
\hline
\end{tabular}

* For details of fat sources, see Table 2. 
Table 4. Phospholipid fatty acids (PL-FA; $\mathrm{g} / 100 \mathrm{~g}$ total fatty acids) of the liver of chicks as affected by age (n 68-74), dietary $\alpha$-tocopherol concentration ( $\mathrm{n} 46-49)$ and dietary fatty acid type (n 45-49)

(Values are means with their standard errors for all chicks to whom the characteristic applied)

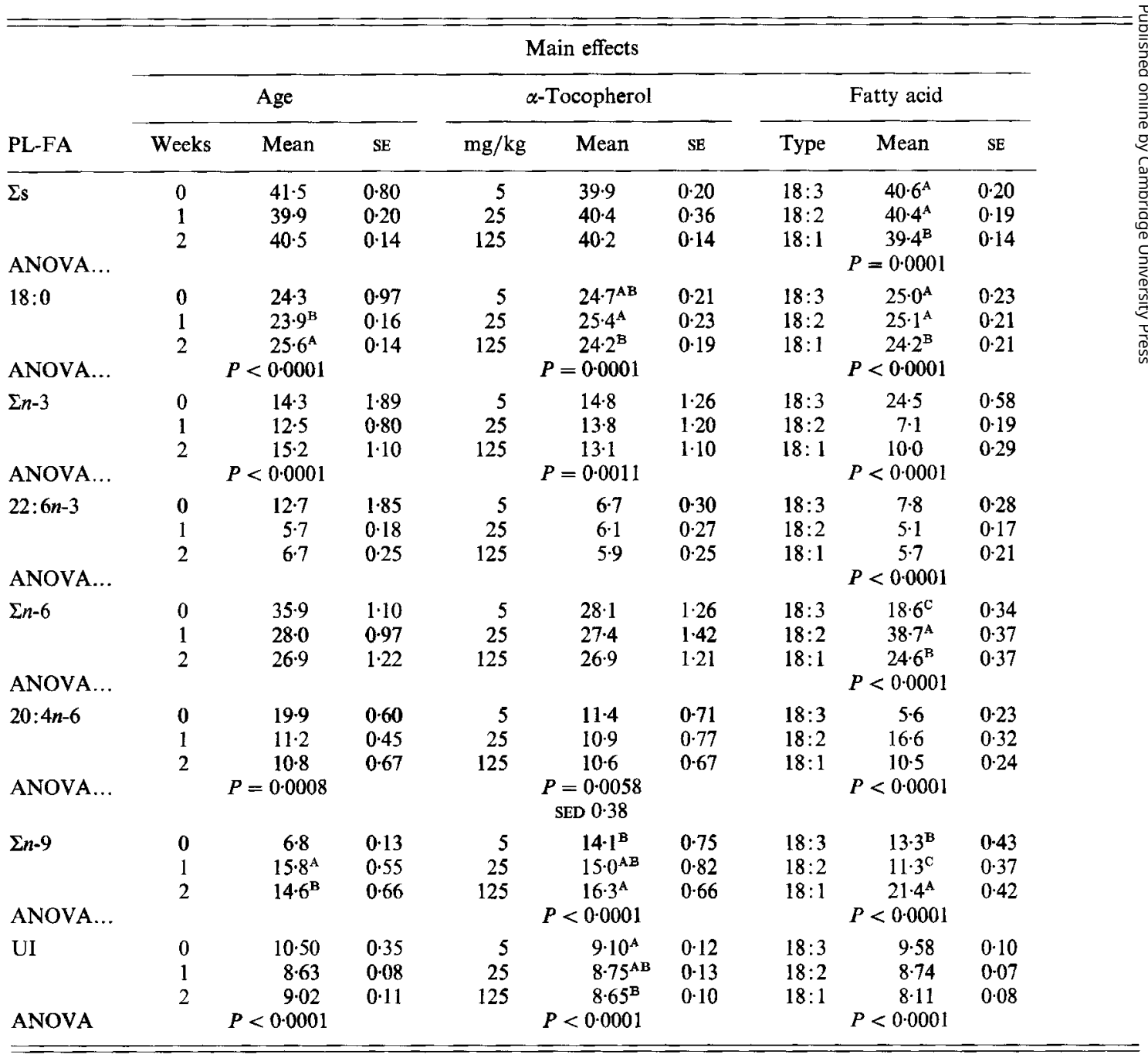

$\Sigma$ s, sum of saturated PL-FA; $\Sigma n-3$, sum of $n-3$ PL-FA; $\Sigma n-6$, sum of $n-6$ PL-FA; $\Sigma n-9$, sum of $n-9$ PL-FA; UI, unsaturation index.

$A, B, C$ Mean values (main effects) not sharing a common superscript letter were significantly different $(P<0.01$, Student-Newman-Keuls).

$\mathrm{K}_{2} \mathrm{CO}_{3}(60 \mathrm{~g} / \mathrm{l})$. A $2 \mu \mathrm{l}$ portion of the supernatant fraction was analysed for fatty acid methyl esters by splitless injection on a Varian 3400 gas chromatograph (Varian, Darmstadt, Germany) equipped with a $30 \mathrm{~m}$ Supelcowax 10 fused silica capillary column $(0.32 \mathrm{~mm}$ i.d.; $0.5 \mu \mathrm{m}$ coating; Supelco, Deisenhofen, Germany). He gas (15 psi, $1.5 \mathrm{ml} / \mathrm{min}$ ) served as carrier gas. Injection temperature was $250^{\circ}$, and the temperature of the flame ionization detector was $260^{\circ}$. The oven temperature was kept at $100^{\circ}$ for 5 min, 
Table 4 -cont.

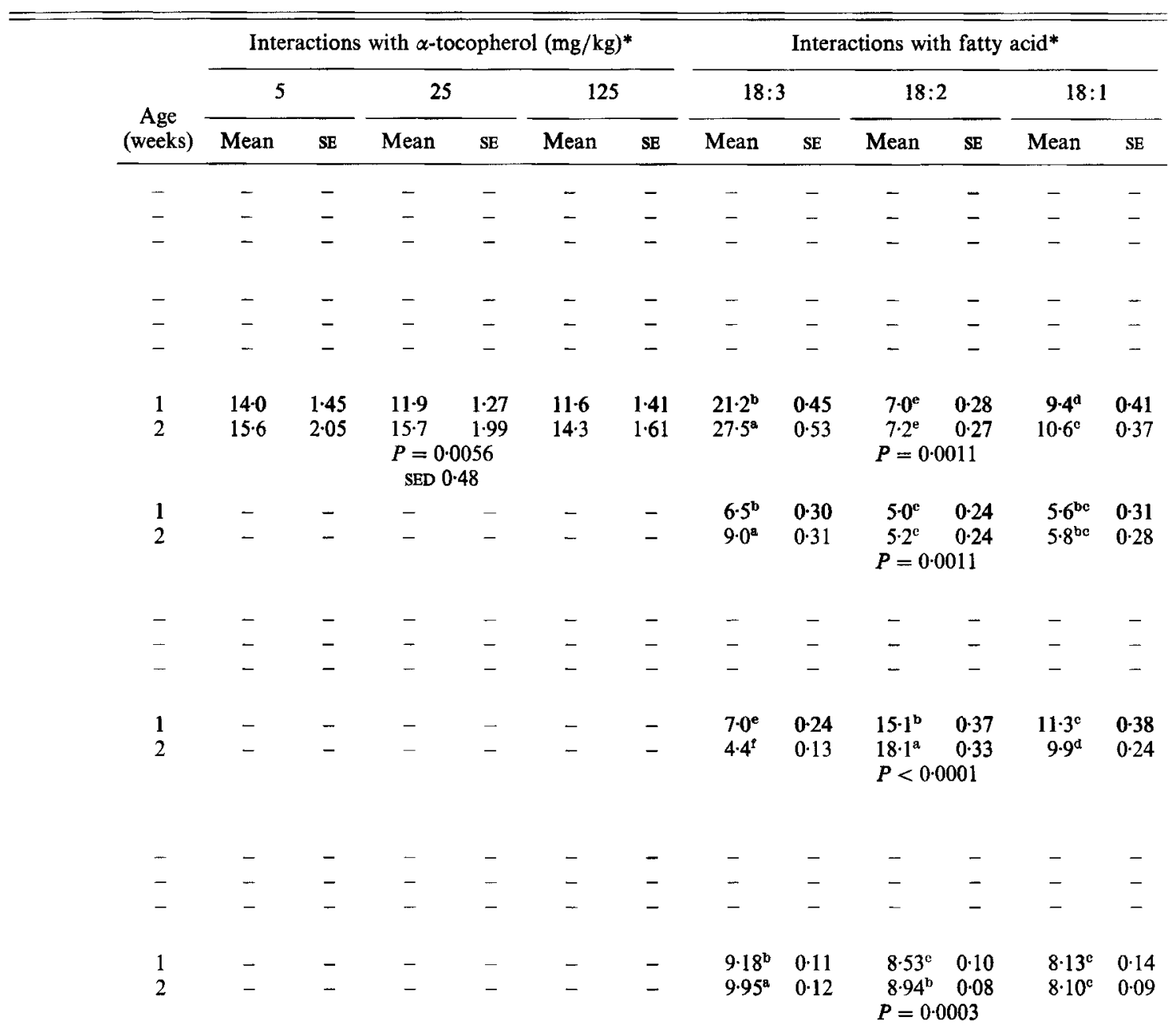

$a, b, c, d, e, t$ Mean values (interactions) not sharing a common superscript letter were significantly different $(P<0.01$, Student-Newman-Keuls).

* For age $\times$ tocopherol, $n$ 23-27; for age $\times$ fat, $n$ 21-25.

followed by an increase of $30^{\circ} / \mathrm{min}$ to reach $190^{\circ}$. The temperature was maintained for $5 \mathrm{~min}$ and then increased further up to $240^{\circ}$ at $5^{\circ} / \mathrm{min}$. Peaks were identified by relative retention times in comparison with commercially available standards. Detection limit was $0.5 \mathrm{ng}$ fatty acid on-column. The inter-assay $\mathrm{CV}(n 6)$ were $<5.2 \%$ and $<10.6 \%$ for fatty acids representing more than $2 \mathrm{~g}$ and $0.5 \mathrm{~g}$ respectively, per $100 \mathrm{~g}$ total fatty acids. Results given in $\mathrm{g} / 100 \mathrm{~g}$ total fatty acids were calculated using the internal standard method of the Apex data system, version 2.04 (Autochrom Inc., Milford, MA, USA). The sum of the 
Table 5. Phospholipid fatty acids ( $P L-F A ; g / 100 \mathrm{~g}$ total fatty acids) of the cerebrum of chicks as affected by age (n 68-74), dietary $\alpha$-tocopherol concentration (n 46-49) and dietary fatty acid type (n 45-49)

(Values are means with their standard errors)

\begin{tabular}{|c|c|c|c|c|c|c|c|c|c|}
\hline \multirow[b]{3}{*}{ PL-FA } & \multicolumn{9}{|c|}{ Main effects } \\
\hline & \multicolumn{3}{|c|}{ Age } & \multicolumn{3}{|c|}{$\alpha$-Tocopherol } & \multicolumn{3}{|c|}{ Fatty acid } \\
\hline & Weeks & Mean & $\mathbf{S E}$ & $\mathrm{mg} / \mathrm{kg}$ & Mean & SE & Type & Mean & $\mathrm{SE}$ \\
\hline$\Sigma s$ & $\begin{array}{l}0 \\
1 \\
2\end{array}$ & $\begin{array}{l}48 \cdot 7 \\
47 \cdot 4 \\
46 \cdot 8\end{array}$ & $\begin{array}{l}0.92 \\
0.28 \\
0.33\end{array}$ & $\begin{array}{r}5 \\
25 \\
125\end{array}$ & $\begin{array}{l}47.6 \\
46.8 \\
46.9\end{array}$ & $\begin{array}{l}0.37 \\
0.48 \\
0.25\end{array}$ & $\begin{array}{l}18: 3 \\
18: 2 \\
18: 1\end{array}$ & $\begin{array}{l}47.9 \\
46 \cdot 9 \\
46.5\end{array}$ & $\begin{array}{l}0.37 \\
0.35 \\
0.38\end{array}$ \\
\hline ANOVA... & $\begin{array}{l}0 \\
1 \\
2\end{array}$ & $\begin{array}{ll}18 \cdot 3 \\
21 \cdot 1^{\mathrm{B}} \\
21 \cdot 7^{\mathrm{A}} \\
P=0 \cdot 0001\end{array}$ & $\begin{array}{l}0.46 \\
0 \cdot 11 \\
0 \cdot 11\end{array}$ & $\begin{array}{r}5 \\
25 \\
125\end{array}$ & $\begin{array}{c}21 \cdot 6^{\mathrm{A}} \\
21 \cdot 6^{\mathrm{A}} \\
21 \cdot 0^{\mathrm{B}} \\
P=0.0001\end{array}$ & $\begin{array}{l}0.14 \\
0.12 \\
0.13\end{array}$ & $\begin{array}{l}18: 3 \\
18: 2 \\
18: 1\end{array}$ & $\begin{array}{l}21 \cdot 3 \\
21 \cdot 6 \\
21 \cdot 4\end{array}$ & $\begin{array}{l}0.15 \\
0.14 \\
0.12\end{array}$ \\
\hline ANOVA... & $\begin{array}{l}0 \\
1 \\
2\end{array}$ & $\begin{array}{l}19 \cdot 9 \\
18 \cdot 3 \\
18 \cdot 6\end{array}$ & $\begin{array}{l}1.01 \\
0.34 \\
0.46\end{array}$ & $\begin{array}{r}5 \\
25 \\
125\end{array}$ & $\begin{array}{l}18.6 \\
18 \cdot 2 \\
18.5\end{array}$ & $\begin{array}{l}0.63 \\
0.47 \\
0.42\end{array}$ & $\begin{array}{l}18: 3 \\
18: 2 \\
18: 1\end{array}$ & $\begin{array}{c}21 \cdot 6^{\mathrm{A}} \\
16 \cdot 3^{\mathrm{B}} \\
17 \cdot 4^{\mathrm{B}} \\
P<0 \cdot 0001\end{array}$ & $\begin{array}{l}0.47 \\
0.32 \\
0.34\end{array}$ \\
\hline $22: 6 n-3$ & $\begin{array}{l}0 \\
1 \\
2\end{array}$ & $\begin{array}{l}18 \cdot 6 \\
16 \cdot 7 \\
16 \cdot 3\end{array}$ & $\begin{array}{l}1 \cdot 01 \\
0 \cdot 28 \\
0 \cdot 29\end{array}$ & $\begin{array}{r}5 \\
25 \\
125\end{array}$ & $\begin{array}{l}16 \cdot 3 \\
16 \cdot 4 \\
16 \cdot 6\end{array}$ & $\begin{array}{l}0.40 \\
0.36 \\
0.31\end{array}$ & $\begin{array}{l}18: 3 \\
18: 2 \\
18: 1\end{array}$ & $\begin{array}{c}18 \cdot 0^{\mathrm{A}} \\
15 \cdot 4^{\mathrm{B}} \\
16 \cdot 1^{\mathrm{B}} \\
P<0 \cdot 0001\end{array}$ & $\begin{array}{l}0 \cdot 32 \\
0 \cdot 30 \\
0 \cdot 32\end{array}$ \\
\hline ANOVA... & $\begin{array}{l}0 \\
1 \\
2\end{array}$ & $\begin{array}{c}15 \cdot 0 \\
15 \cdot 3 \\
14 \cdot 7 \\
P=0.0002\end{array}$ & $\begin{array}{l}1 \cdot 12 \\
0 \cdot 22 \\
0 \cdot 41\end{array}$ & $\begin{array}{r}5 \\
25 \\
125\end{array}$ & $\begin{array}{l}15 \cdot 0 \\
15 \cdot 0 \\
14 \cdot 9\end{array}$ & $\begin{array}{l}0.45 \\
0.41 \\
0.40\end{array}$ & $\begin{array}{l}18: 3 \\
18: 2 \\
18: 1\end{array}$ & $\begin{array}{c}12 \cdot 2 \\
18 \cdot 3 \\
14 \cdot 4 \\
P<0.0001\end{array}$ & $\begin{array}{l}0.21 \\
0.22 \\
0 \cdot 14\end{array}$ \\
\hline ANOVA... & $\begin{array}{l}0 \\
1 \\
2\end{array}$ & $\begin{array}{c}9.9 \\
10 \cdot 3 \\
9 \cdot 7 \\
P<0.0001\end{array}$ & $\begin{array}{l}0.13 \\
0.12 \\
0.23\end{array}$ & $\begin{array}{r}5 \\
25 \\
125\end{array}$ & $\begin{array}{r}10 \cdot 0 \\
10 \cdot 0 \\
9 \cdot 9\end{array}$ & $\begin{array}{l}0.27 \\
0.22 \\
0 \cdot 28\end{array}$ & $\begin{array}{l}18: 3 \\
18: 2 \\
18: 1\end{array}$ & $\begin{array}{c}8.4 \\
11.7 \\
9.7 \\
P<0.0001\end{array}$ & $\begin{array}{l}0 \cdot 14 \\
0 \cdot 11 \\
0 \cdot 09\end{array}$ \\
\hline ANOVA... & $\begin{array}{l}0 \\
1 \\
2\end{array}$ & $\begin{array}{c}12 \cdot 4 \\
13 \cdot 8 \\
14 \cdot 4 \\
P=0.0012\end{array}$ & $\begin{array}{l}0.12 \\
0.16 \\
0.23\end{array}$ & $\begin{array}{r}5 \\
25 \\
125\end{array}$ & $\begin{array}{c}13 \cdot 7 \\
14 \cdot 4 \\
14 \cdot 3 \\
P=0.0015 \\
\text { SED } 0 \cdot 20\end{array}$ & $\begin{array}{l}0.27 \\
0.24 \\
0.25\end{array}$ & $\begin{array}{l}18: 3 \\
18: 2 \\
18: 1\end{array}$ & $\begin{array}{c}13.2 \\
13.2 \\
16.1 \\
P<0.0001\end{array}$ & $\begin{array}{l}0 \cdot 13 \\
0 \cdot 15 \\
0 \cdot 20\end{array}$ \\
\hline UI & $\begin{array}{l}0 \\
1 \\
2\end{array}$ & $\begin{array}{l}9.52 \\
9 \cdot 06 \\
9.04\end{array}$ & $\begin{array}{l}0.27 \\
0.08 \\
0.08\end{array}$ & $\begin{array}{r}5 \\
25 \\
125\end{array}$ & $\begin{array}{l}9.00 \\
9.02 \\
9 \cdot 12\end{array}$ & $\begin{array}{l}0.11 \\
0.11 \\
0.08\end{array}$ & $\begin{array}{l}18: 3 \\
18: 2 \\
18: 1\end{array}$ & $\begin{array}{l}9 \cdot 12 \\
9.09 \\
8.94\end{array}$ & $\begin{array}{l}0 \cdot 10 \\
0 \cdot 10 \\
0 \cdot 10\end{array}$ \\
\hline
\end{tabular}

$\Sigma$ s, sum of saturated PL-FA; $\Sigma n-3$, sum of $n-3$ PL-FA; $\Sigma n-6$, sum of $n-6$ PL-FA; $\Sigma n-9$, sum of $n-9$ PL-FA; UI, unsaturation index.

A, B Mean values (main effects) not sharing a common superscript letter were significantly different $(P<0 \cdot 01$, Student-Newman-Keuls).

saturated phospholipid fatty acids $(\Sigma s)$ included 12:0, 14:0, 16:0, 20:0, 22:0 and 24:0 besides the 18:0 shown in the tables. The sum of the $n-3$ fatty acids $(\Sigma n-3)$ included 18:3, $20: 3,20: 5$ and $22: 5$ besides the $22: 6$ shown in the tables. The sum of the $n-6$ fatty acids $(\Sigma n-6)$ included $18: 2,18: 3,20: 2,20: 3,22: 4$ and $22: 5$ besides the $20: 4$ shown in the tables. The sum of the phospholipid $n-9$ fatty acids $(\Sigma n-9)$ included 16:1, 18:1, 20:1, 20:3 and 22:1. Dimethyl acetals from plasmalogens are not included in the sums of saturates and monoenes. An unsaturation index (UI) was calculated from the unsaturated fatty acids. It 
Table 5-cont.

\begin{tabular}{|c|c|c|c|c|c|c|}
\hline \multirow{3}{*}{$\begin{array}{c}\text { Age } \\
\text { (weeks) }\end{array}$} & \multicolumn{6}{|c|}{ Interactions with fatty acid* } \\
\hline & \multicolumn{2}{|c|}{$18: 3$} & \multicolumn{2}{|c|}{$18: 2$} & \multicolumn{2}{|c|}{$18: 1$} \\
\hline & Mean & SE & Mean & $\mathbf{S E}$ & Mean & SE \\
\hline- & - & - & - & - & - & - \\
\hline- & - & - & - & - & - & - \\
\hline- & - & - & - & - & - & - \\
\hline- & - & - & - & - & - & - \\
\hline- & - & - & - & - & - & - \\
\hline- & - & - & - & - & - & - \\
\hline- & - & - & - & - & - & - \\
\hline- & - & - & - & - & - & - \\
\hline- & - & - & - & - & - & - \\
\hline - & - & - & - & - & - & - \\
\hline- & - & - & - & - & - & - \\
\hline- & - & - & - & - & - & - \\
\hline 1 & $13 \cdot 5^{\mathrm{d}}$ & $0 \cdot 15$ & $17 \cdot 3^{b}$ & $0 \cdot 24$ & $15 \cdot 1^{\mathrm{e}}$ & $0 \cdot 18$ \\
\hline \multirow[t]{2}{*}{2} & $10 \cdot 9^{\mathrm{e}}$ & 0.12 & $19 \cdot 2^{\mathrm{a}}$ & $0 \cdot 23$ & $13 \cdot 9^{d}$ & $0 \cdot 15$ \\
\hline & \multicolumn{6}{|c|}{$P<0.0001$} \\
\hline 1 & $9 \cdot 3^{d}$ & 0.09 & $11 \cdot 4^{b}$ & $0 \cdot 14$ & $10 \cdot 2^{c}$ & $0 \cdot 10$ \\
\hline \multirow[t]{2}{*}{2} & $7 \cdot 6^{\mathrm{e}}$ & 0.09 & $12 \cdot 1^{8}$ & 0.13 & $9 \cdot 2^{\mathrm{d}}$ & 0.08 \\
\hline & \multicolumn{6}{|c|}{$P<0.0001$} \\
\hline 1 & $13 \cdot 0^{\mathrm{c}}$ & 0.18 & $13 \cdot 4^{\mathrm{c}}$ & $0 \cdot 24$ & $15 \cdot 1^{\mathrm{b}}$ & $0 \cdot 19$ \\
\hline \multirow[t]{2}{*}{2} & $13 \cdot 4^{c}$ & $0 \cdot 18$ & $13 \cdot 1^{\mathrm{C}}$ & $0 \cdot 19$ & $16 \cdot 9^{a}$ & $0 \cdot 24$ \\
\hline & \multicolumn{6}{|c|}{$P<0.0001$} \\
\hline- & - & - & - & - & - & - \\
\hline- & - & - & - & - & - & - \\
\hline- & - & - & - & - & - & - \\
\hline- & - & - & - & - & - & - \\
\hline- & - & - & - & - & - & - \\
\hline
\end{tabular}

a.b,e,d,e Mean values (interactions) not sharing a common superscript letter were significantly different $(P<0.01$, Student-Newman-Keuls).

* For age $\times$ fat interactions, $n$ 21-25.

is defined as the sum of the products of \% total fatty acids and number of double bonds, divided by the number of $\mathrm{C}$ atoms of each fatty acid. Division by chain length was done to correct for the different ratio between double and single bonds in each fatty acid.

Statistical analysis was done by 3-factorial ANOVA for the factors age, dietary $\alpha$ tocopherol and fat (Friedman, 1986). The grouping due to the fact that the chicks on the same diet were kept in the same cage was not considered. When the Bartlett-Box $F$ test indicated variance inhomogeneity the data were transformed logarithmically. Main effects and interactions were assumed to be significant at $P<0 \cdot 01$. In this case means were tested 
Table 6. Phospholipid fatty acids $(P L-F A ; g / 100 \mathrm{~g}$ total fatty acids) of the cerebellum of chicks as affected by age (n 69-75), dietary $\alpha$-tocopherol concentration (n 68-75) and dietary fatty acid type (n 70-74)

(Values are means with their standard errors)

\begin{tabular}{|c|c|c|c|c|c|c|c|c|c|}
\hline \multirow[b]{3}{*}{ PL-FA } & \multicolumn{9}{|c|}{ Main effects } \\
\hline & \multicolumn{3}{|c|}{ Age } & \multicolumn{3}{|c|}{$\alpha$-Tocopherol } & \multicolumn{3}{|c|}{ Fatty acid } \\
\hline & Weeks & Mean & SE & $\mathrm{mg} / \mathrm{kg}$ & Mean & $\mathrm{SE}$ & Type & Mean & SE \\
\hline ANOVA... & $\begin{array}{l}0 \\
1 \\
2 \\
3\end{array}$ & $\begin{aligned} & 46 \cdot 9 \\
& 43 \cdot 4 \\
& 42 \cdot 5 \\
& 45 \cdot 0 \\
&=0.0001\end{aligned}$ & $\begin{array}{l}1.33 \\
0.25 \\
0.26 \\
0.28\end{array}$ & $\begin{array}{r}5 \\
25 \\
125 \\
-\end{array}$ & $\begin{array}{c}43 \cdot 8 \\
43 \cdot 4 \\
43 \cdot 5 \\
-\end{array}$ & $\begin{array}{c}0.29 \\
0.26 \\
0.31 \\
-\end{array}$ & $\begin{array}{l}18: 3 \\
18: 2 \\
18: 1 \\
-\end{array}$ & $\begin{array}{c}43 \cdot 8 \\
43 \cdot 3 \\
43 \cdot 8 \\
-\end{array}$ & $\begin{array}{c}0 \cdot 46 \\
0 \cdot 27 \\
0 \cdot 31 \\
-\end{array}$ \\
\hline $18: 0$ & $\begin{array}{l}0 \\
1 \\
2 \\
3\end{array}$ & $\begin{array}{l}17 \cdot 0 \\
19 \cdot 1 \\
19 \cdot 3 \\
19 \cdot 3\end{array}$ & $\begin{array}{l}0.35 \\
0.17 \\
0.41 \\
0.11\end{array}$ & $\begin{array}{r}5 \\
25 \\
125 \\
-\end{array}$ & $\begin{array}{l}19 \cdot 7 \\
19 \cdot 0 \\
19 \cdot 0 \\
-\end{array}$ & $\begin{array}{l}0.09 \\
0.41 \\
0.12 \\
-\end{array}$ & $\begin{array}{l}18: 3 \\
18: 2 \\
18: 1 \\
-\end{array}$ & $\begin{array}{c}19 \cdot 0 \\
19 \cdot 3 \\
19 \cdot 5 \\
-\end{array}$ & $\begin{array}{c}0.10 \\
0 \cdot 12 \\
0.44 \\
-\end{array}$ \\
\hline ANOVA... & $\begin{array}{l}0 \\
1 \\
2 \\
3\end{array}$ & $\begin{array}{c}17 \cdot 7 \\
18 \cdot 2 \\
18 \cdot 1 \\
15 \cdot 7 \\
<0.0001\end{array}$ & $\begin{array}{l}1 \cdot 66 \\
0.29 \\
0.42 \\
0.46\end{array}$ & $\begin{array}{r}5 \\
25 \\
125 \\
-\end{array}$ & $\begin{array}{c}17 \cdot 2 \\
17 \cdot 7 \\
17 \cdot 1 \\
-\end{array}$ & $\begin{array}{c}0.38 \\
0.47 \\
0.42 \\
-\end{array}$ & $\begin{array}{l}18: 3 \\
18: 2 \\
18: 1 \\
-\end{array}$ & $\begin{array}{c}20 \cdot 8 \\
15 \cdot 3 \\
15 \cdot 7 \\
-\overline{-} 0.000 .\end{array}$ & $\begin{array}{l}0.29 \\
0.29 \\
0.30 \\
-\end{array}$ \\
\hline ANOVA... & $\begin{array}{l}0 \\
1 \\
2 \\
3\end{array}$ & $\begin{array}{c}16 \cdot 3 \\
15 \cdot 5^{\mathrm{A}} \\
14-7^{\mathrm{A}} \\
12 \cdot 7^{\mathrm{B}} \\
<0 \cdot 0001\end{array}$ & $\begin{array}{l}1.69 \\
0.23 \\
0.27 \\
0.27\end{array}$ & $\begin{array}{r}5 \\
25 \\
125 \\
-\end{array}$ & $\begin{array}{c}14 \cdot 6 \\
14 \cdot 5 \\
13 \cdot 8 \\
-\end{array}$ & $\begin{array}{l}0.29 \\
0.29 \\
0.30 \\
-\end{array}$ & $\begin{array}{c}18: 3 \\
18: 2 \\
18: 1 \\
-\end{array}$ & $\begin{array}{c}15 \cdot 7^{\mathrm{A}} \\
13 \cdot 5^{\mathrm{B}} \\
13 \cdot 6^{\mathrm{B}} \\
-\overline{-} \\
<0 \cdot 000\end{array}$ & $\begin{array}{c}0.26 \\
0.27 \\
0.27 \\
-\end{array}$ \\
\hline ANOVA... & $\begin{array}{l}0 \\
1 \\
2 \\
3\end{array}$ & $\begin{array}{c}14.6 \\
12.9 \\
11.9 \\
12.9 \\
<0.0001\end{array}$ & $\begin{array}{l}0.39 \\
0.36 \\
0.38 \\
0.50\end{array}$ & $\begin{array}{r}5 \\
25 \\
125 \\
-\end{array}$ & $\begin{array}{l}12 \cdot 7 \\
12 \cdot 5 \\
12 \cdot 4 \\
-\end{array}$ & $\begin{array}{l}0.36 \\
0.42 \\
0.38 \\
-\end{array}$ & $\begin{array}{l}18: 3 \\
18: 2 \\
18: 1 \\
-\end{array}$ & $\begin{array}{c}9.4 \\
16.4 \\
11.9 \\
- \\
<0.000\end{array}$ & $\begin{array}{c}0.16 \\
0.22 \\
0.12 \\
-\end{array}$ \\
\hline ANOVA... & $\begin{array}{l}0 \\
1 \\
2 \\
3\end{array}$ & $\begin{array}{c}8 \cdot 1 \\
7 \cdot 0 \\
6 \cdot 2 \\
6 \cdot 7 \\
<0.0001\end{array}$ & $\begin{array}{l}0.33 \\
0.08 \\
0.17 \\
0.24\end{array}$ & $\begin{array}{r}5 \\
25 \\
125 \\
-\end{array}$ & $\begin{array}{l}6.7 \\
6.6 \\
6.6 \\
-\end{array}$ & $\begin{array}{l}0.17 \\
0.20 \\
0.18 \\
-\end{array}$ & $\begin{array}{c}18: 3 \\
18: 2 \\
18: 1 \\
-\end{array}$ & $\begin{array}{c}5 \cdot 1 \\
8 \cdot 3 \\
6 \cdot 4 \\
-1 \\
<0.000\end{array}$ & $\begin{array}{l}0.10 \\
0.10 \\
0.08 \\
-\end{array}$ \\
\hline ANOVA... & $\begin{array}{l}0 \\
1 \\
2 \\
3\end{array}$ & $\begin{array}{c}17 \cdot 1 \\
19 \cdot 6 \\
21 \cdot 1 \\
20 \cdot 3 \\
<0 \cdot 0001\end{array}$ & $\begin{array}{l}0.25 \\
0.16 \\
0.26 \\
0.29\end{array}$ & $\begin{array}{r}5 \\
25 \\
125 \\
-\end{array}$ & $\begin{array}{c}20 \cdot 2 \\
20 \cdot 2 \\
20 \cdot 6 \\
-\end{array}$ & $\begin{array}{c}0.24 \\
0.27 \\
0.26 \\
-\end{array}$ & $\begin{array}{c}18: 3 \\
18: 2 \\
18: 1 \\
-\end{array}$ & $\begin{array}{c}20 \cdot 1 \\
19 \cdot 0 \\
22 \cdot 0 \\
- \\
<0 \cdot 000\end{array}$ & $\begin{array}{c}0.19 \\
0.21 \\
0.26 \\
-\end{array}$ \\
\hline ANOVA... & $\begin{array}{l}0 \\
1 \\
2 \\
3\end{array}$ & $\begin{array}{c}8.63 \\
8.65 \\
8.55 \\
8.02 \\
<0.0001\end{array}$ & $\begin{array}{l}0.42 \\
0.06 \\
0.07 \\
0.07\end{array}$ & $\begin{array}{r}5 \\
25 \\
125 \\
-\end{array}$ & $\begin{array}{l}8 \cdot 38 \\
8 \cdot 48 \\
8 \cdot 36 \\
-\end{array}$ & $\begin{array}{c}0.06 \\
0.08 \\
0.09 \\
-\end{array}$ & $\begin{array}{c}18: 3 \\
18: 2 \\
18: 1 \\
-\end{array}$ & $\begin{array}{r}8 \cdot 57^{\mathrm{A}} \\
8 \cdot 51^{\mathrm{A}} \\
8 \cdot 12^{\mathrm{B}} \\
- \\
<0.0001\end{array}$ & $\begin{array}{c}0.07 \\
0.06 \\
0.08 \\
-\end{array}$ \\
\hline
\end{tabular}

$\Sigma$ s, sum of saturated PL-FA; $\Sigma n-3$, sum of $n-3$ PL-FA; $\Sigma n-6$, sum of $n-6$ PL-FA; $\Sigma n-9$, sum of $n-9$ PL-FA; UI, unsaturation index.

A, B Mean values (main effects) not sharing a common superscript letter were significantly different $(P<0.01$, Student-Newman-Keuls). 
Table 6-cont.

\begin{tabular}{|c|c|c|c|c|c|c|c|c|c|c|c|c|}
\hline \multirow{3}{*}{$\begin{array}{c}\text { Age } \\
\text { (weeks) }\end{array}$} & \multicolumn{6}{|c|}{ Interactions with $\alpha$-tocopherol $(\mathrm{mg} / \mathrm{kg})^{*}$} & \multicolumn{6}{|c|}{ Interactions with fatty acid* } \\
\hline & \multicolumn{2}{|l|}{5} & \multicolumn{2}{|l|}{25} & \multicolumn{2}{|c|}{125} & \multicolumn{2}{|c|}{$18: 3$} & \multicolumn{2}{|c|}{$18: 2$} & \multicolumn{2}{|c|}{$18: 1$} \\
\hline & Mean & SE & Mean & $\mathrm{SE}$ & Mean & $\mathrm{SE}$ & Mean & $\mathrm{SE}$ & Mean & SE & Mean & SE \\
\hline 1 & $43 \cdot 8^{\mathrm{b}}$ & 0.35 & $43 \cdot 5^{\mathrm{b}}$ & 0.52 & $42 \cdot 8^{\mathrm{b}}$ & 0.43 & - & - & - & - & - & - \\
\hline 2 & $43 \cdot 3^{b}$ & 0.40 & $42 \cdot 6^{\mathrm{b}}$ & 0.51 & $41 \cdot 7^{\mathrm{b}}$ & 0.38 & - & _- & - & - & - & - \\
\hline \multirow[t]{2}{*}{3} & $44 \cdot 6^{\mathrm{ab}}$ & 0.43 & $44 \cdot 1^{\text {ab }}$ & 0.31 & $46 \cdot 2^{\mathrm{a}}$ & 0.58 & - & - & - & - & - & - \\
\hline & & & \multicolumn{2}{|c|}{$P=0.0005$} & & & & & & & & \\
\hline - & - & - & - & - & - & - & - & - & - & - & - & - \\
\hline - & - & - & - & - & - & - & - & - & - & - & - & - \\
\hline - & - & - & - & - & - & - & - & - & - & - & - & - \\
\hline 1 & - & - & - & - & - & - & $20 \cdot 1^{\mathrm{b}}$ & 0.51 & $17 \cdot 2^{\mathrm{c}}$ & 0.35 & $17 \cdot 2^{\mathrm{c}}$ & 0.35 \\
\hline 2 & - & - & - & - & - & - & $22 \cdot 1^{\mathrm{a}}$ & 0.44 & $16 \cdot 0^{c}$ & 0.34 & $15 \cdot 8^{\mathrm{c}}$ & 0.53 \\
\hline \multirow[t]{2}{*}{3} & - & - & - & - & - & - & $20 \cdot 1^{\mathrm{b}}$ & 0.48 & $12 \cdot 5^{\mathrm{e}}$ & 0.24 & $14 \cdot 2^{\mathrm{d}}$ & 0.48 \\
\hline & & & & & & & & & \multicolumn{2}{|c|}{$P<0.0001$} & & \\
\hline - & - & - & - & - & - & - & - & - & - & - & - & - \\
\hline - & - & - & - & - & - & - & - & - & - & - & - & - \\
\hline - & - & - & - & - & - & - & - & - & - & - & - & - \\
\hline 1 & - & - & - & - & - & - & $11 \cdot 2^{\mathrm{e}}$ & 0.14 & $14 \cdot 7^{\mathrm{e}}$ & 0.15 & $12 \cdot 6^{\mathrm{d}}$ & 0.13 \\
\hline 2 & - & - & - & - & - & - & $8 \cdot 6^{t}$ & 0.12 & $16 \cdot 1^{\mathrm{b}}$ & $0 \cdot 18$ & $11 \cdot 0^{\mathrm{e}}$ & 0.13 \\
\hline \multirow[t]{2}{*}{3} & - & - & - & - & - & - & $8 \cdot 6^{\mathrm{t}}$ & 0.14 & $18 \cdot 7^{a}$ & 0.24 & $12 \cdot 0^{\alpha}$ & 0.22 \\
\hline & & & & & & & & & \multicolumn{2}{|c|}{$P<0.0001$} & & \\
\hline 1 & - & - & - & - & - & - & $6 \cdot 3^{\mathrm{e}}$ & 0.07 & $7 \cdot 7^{\mathrm{c}}$ & 0.08 & $7 \cdot 0^{\mathrm{d}}$ & 0.08 \\
\hline 2 & - & - & - & - & - & - & $4 \cdot 7^{8}$ & 0.06 & $8 \cdot 1^{\mathrm{b}}$ & $0 \cdot 10$ & $5 \cdot 9^{f}$ & 0.07 \\
\hline \multirow[t]{2}{*}{3} & - & - & - & - & - & - & $4 \cdot 5^{8}$ & 0.08 & $9 \cdot 3^{\mathrm{a}}$ & $0 \cdot 14$ & $6 \cdot 5^{\mathrm{e}}$ & 0.14 \\
\hline & & & & & & & & & \multicolumn{2}{|c|}{$P<0.0001$} & & \\
\hline 1 & - & - & - & - & - & - & $19 \cdot 2^{\text {de }}$ & 0.23 & $19 \cdot 1^{\mathrm{de}}$ & 0.29 & $20 \cdot 6^{\text {bed }}$ & 0.20 \\
\hline 2 & - & - & - & - & - & - & $20 \cdot 1^{\text {cd }}$ & 0.26 & $19 \cdot 7^{\mathrm{co}}$ & 0.28 & $23 \cdot 6^{\mathbf{a}}$ & 0.37 \\
\hline \multirow[t]{2}{*}{3} & - & - & - & _- & - & - & $20.8^{\mathrm{be}}$ & 0.41 & $18 \cdot 1^{e}$ & 0.46 & $21 \cdot 7^{b}$ & 0.34 \\
\hline & & & & & & & & & \multicolumn{2}{|c|}{$P<0.0001$} & & \\
\hline 1 & $8 \cdot 61^{\mathrm{ab}}$ & 0.11 & $8 \cdot 64^{\mathrm{ab}}$ & $0 \cdot 12$ & $8 \cdot 70^{\mathrm{a}}$ & $0 \cdot 11$ & - & - & - & - & - & - \\
\hline 2 & $8 \cdot 39^{\mathrm{ab}}$ & $0 \cdot 11$ & $8 \cdot 59^{\mathrm{ab}}$ & 0.16 & $8 \cdot 65^{\mathrm{ab}}$ & $0 \cdot 10$ & - & - & - & - & - & - \\
\hline \multirow[t]{2}{*}{3} & $8.08^{\mathrm{bc}}$ & 0.09 & & & $7 \cdot 72^{\mathrm{e}}$ & 0.14 & - & - & - & - & - & - \\
\hline & & & \multicolumn{2}{|c|}{$P=0.008$} & & & & & & & & \\
\hline
\end{tabular}

${ }^{a-g}$ Mean values (interactions) not sharing a common superscript letter were significantly different $(P<0.01$, Student-Newman-Keuls).

* For age $\times$ tocopherol interactions, $n$ 19-27; for age $\times$ fat interactions, $n$ 22-27. 
for significant differences ( $99 \%$ confidence level) by the Student-Newman-Keuls test. When the Student-Newman-Keuls test failed to show significant differences between means despite significant main effects or interactions according to the ANOVA, the standard error of difference between any two means (SED) was calculated. Data from the newly hatched chicks $(n)$ were not included.

\section{RESULTS}

Body-weight gain and feed conversion values did not indicate any feeding effects (results not shown). The bias produced by keeping the chicks on the same diets in the same cages is considered to be minimal, because the groups were randomly allocated to the treatments and the environmental conditions in the fully climatized room were the same for all groups. Furthermore, the basal diet was prepared in one batch for the whole trial. Thus, possible effects on analysis should be negligible. Beginning at day 9, clinical signs of NE occurred in four out of twenty-nine animals from the group that received the $\alpha$-tocopherol-deficient, linoleic acid-rich diet.

\section{Phospholipid fatty acid composition of the liver}

Dietary fatty acids greatly affected the phospholipid fatty acid composition of the liver (Table 4). Oleic acid-rich fat led to a significantly lower proportion of $\Sigma$ s in liver phospholipids in comparison with linolenic and linoleic-acid feeding. The influence of $\alpha$ tocopherol on $\Sigma$ s was inconsistent, because 16:0 (not shown) and 18:0 responded differently. Dietary linolenic acid elevated $\Sigma n-3$ from $14.3 \mathrm{~g} / 100 \mathrm{~g}$ in the 1-d-old chick up to $27.5 \mathrm{~g} / 100 \mathrm{~g}$ total fatty acids after 2 weeks. This was attributable to $20: 5 n-3$ $(0.5 \rightarrow 12 \mathrm{~g} / 100 \mathrm{~g}$, results not shown). On the other hand $22: 6 n-3$ decreased. $\Sigma n-3$ was influenced by vitamin E. Feeding linoleic acid to the animals increased $\Sigma n-6$ in comparison with the level of the newly hatched chick. $\Sigma n-6$ was not affected by $\alpha$-tocopherol; $20: 4 n-6$ was the $n-6$ fatty acid with the most distinctive changes during growth. $\alpha$-Tocopherol significantly affected $20: 4 n-6$. This was independent of the fat source supplied. $\Sigma n-9$ varied between $11.3 \mathrm{~g} / 100 \mathrm{~g}$ in the linoleic and $21.4 \mathrm{~g} / 100 \mathrm{~g}$ total fatty acids in the oleic acid-rich groups. The $125 \mathrm{mg} \alpha$-tocopherol level led to a higher proportion of $\Sigma n-9$. This was attributable to the increase of 18:1n-9 (not shown) in liver phospholipids. The UI in liver, as a measure of the unsaturation of membrane phospholipids, decreased after hatching to increase again after 2 weeks when linolenic or linoleic acid was fed. The highest values were found in vitamin E-deficient animals and when linolenic acid was fed.

\section{Phospholipid fatty acid composition of the cerebrum}

In the cerebrum the $\Sigma$ s was not affected by dietary conditions and age (Table 5). However 18:0 was reduced by the diet containing $125 \mathrm{mg}$ dietary $\alpha$-tocopherol $/ \mathrm{kg}$. $\Sigma n-3$ in cerebrum was significantly raised by feeding linolenic acid; $22: 6 n-3$, as the relevant fatty acid of this family, was higher in the linolenic acid groups than in those given linolenic or oleic acid. $\Sigma n-6$ was affected by age and dietary fat. Only the linoleic acid-rich diet led to a significant increase of this fatty acid family after 2 weeks. The 20:4n-6 decreased during the first 2 weeks when linolenic or oleic acid was supplied. $\Sigma n-9$ was significantly heightened when oleic acid was the main dietary fatty acid. The cerebral UI was not influenced by the experimental conditions.

\section{Phospholipid fatty acid composition of the cerebellum}

In the cerebellum (Table 6) $\Sigma$ s depended on age, with an increase after 3 weeks. This was most pronounced when $125 \mathrm{mg} \alpha$-tocopherol $/ \mathrm{kg}$ was supplied. Cerebellar $\Sigma n-3$ was significantly heightened by feeding linolenic acid especially after 2 weeks. In the linoleic 
acid groups the $\Sigma n-3$ showed a drastic decrease from $17.2 \mathrm{~g} / 100 \mathrm{~g}$ after 1 week to $12.5 \mathrm{~g} / 100 \mathrm{~g}$ total fatty acids after 3 weeks. This was also valid for the oleic acid groups but to a lesser extent. The level of $22: 5 n-3$ (not shown) was raised from the 1-d-old chick; $22: 6 n-3$, on the other hand, decreased over all groups. Feeding linoleic acid to the animals for 3 weeks led to a rise of $\Sigma n-6$ up to $18.7 \mathrm{~g} / 100 \mathrm{~g}$ total fatty acids in comparison with the level of the newly hatched chick $(14.6 \mathrm{~g} / 100 \mathrm{~g})$. As in liver and cerebrum $\Sigma n-6$ was not affected by $\alpha$-tocopherol; $20: 4 n-6$ decreased after hatching to increase again, when linoleic acid was fed. $\Sigma n-9$ and its individual fatty acids increased after hatching. However there was a drop after 3 weeks within the linoleic and oleic acid groups. Oleic acid feeding led to a significantly lower UI after 3 weeks, when $125 \mathrm{mg} \alpha$-tocopherol $/ \mathrm{kg}$ was supplied. The UI of cerebellar phospholipids (total mean $8 \cdot 40,95 \% \mathrm{CI} 8 \cdot 32,8 \cdot 49$ ) was lower in comparison with cerebrum (UI: 8.83, 95\% CI, 8.69, 8.97) and liver (UI: 9.05, 95\% CI 8.94, 9.16).

\section{DISCUSSION}

Lipid metabolism of the newly hatched chick undergoes a rapid and extensive change (Noble et al. 1993). The residual lipid of the yolk is completely taken up by day 4 after hatching (Chamblee et al. 1992). During this time normal lipid digestion is established. The chicken diets used in the present experiment were designed to be free from very-long-chain fatty acids in order to study the effect of $\alpha$-tocopherol on tissue incorporation of the C18 fatty acids. The experimental conditions should give hints as to the influence of $\alpha$ tocopherol on this process during the first weeks post-hatching.

$\alpha$-Tocopherol (Fuhrmann \& Sallmann, 1995) and tissue fatty acids (Anderson et al. 1989 , 1990, 1992; Lin et al. 1991; Anderson, 1994) of the newly hatched chick are extensively determined by the diet of the laying hen and therefore important for the susceptibility of chicks to NE (Bartov \& Bornstein, 1980). The hens' diet was marginally supplied with $\alpha$ tocopherol in order to produce chickens with low tissue $\alpha$-tocopherol levels. This was intended to initiate early onset of the disease. On the other hand, $5 \mathrm{mg} \alpha$-tocopherol $/ \mathrm{kg}$ was used as the low dose in the chicken diet to avoid high mortality due to encephalomalacia, resulting in the complete loss of the vitamin E-deficient group given linoleic acid. The hens' feed contained $18: 2 n-6$ and $18: 3 n-3$ from soyabean oil together with long-chain $n-3$ fatty acids from fish meal ( $11.2 \mathrm{~g}$ total $n-3 / 100 \mathrm{~g}, n-6: n-31.7)$ leading to $14 \mathrm{~g} / 100 \mathrm{~g} \Sigma n-3$ in liver, $20 \mathrm{~g} / 100 \mathrm{~g} \Sigma n-3$ in cerebrum and $18 \mathrm{~g} / 100 \mathrm{~g} \Sigma n-3$ in cerebellum phospholipids. The hen's diet is responsible for the differences in the fatty acid pattern of our 1-d-old chicks in comparison with that in an earlier experiment by Anderson et al. (1989). The authors investigated the effect of the hen's diet on tissue fatty acids of newly hatched chicks and reported a comparable fatty acid pattern and $n-6: n-3$ in chicks stemming from hens fed on a soyabean-oil diet.

In chicken, $\Sigma$ s of the tissues examined was regulated within a narrow range. However an influence of dietary fat existed in the liver when oleic acid-rich fats were given. $\Sigma$ s was diminished, probably in response to the lower content of polyenoic fatty acids in phospholipids under oleic acid-rich diets. This might help to maintain fluidity of the liver membrane, which is in large part determined by chain length and desaturation of phospholipid fatty acids (Sardesai, 1992). In an earlier study of $n-3$ fatty acid deficiency in chicks Anderson et al. (1990) found that dietary 18:3n-3 was not very effective in raising levels of $20: 5 n-3$ and $22: 6 n-3$ after hatching. This is apparently different to the present results in which high levels of $22: 6 n-3$ in brain were kept constant by feeding $18: 3 n-3$. On the other hand, liver $22: 6 n-3$ fell from $12.7 \mathrm{~g} / 100 \mathrm{~g}$ total fatty acids at hatching to $6.5 \mathrm{~g} / 100 \mathrm{~g}$ after 1 week. This is probably connected with an extensive transfer of longchain $n-3$ fatty acids from liver to brain. However, at least in the rat, brain endothelia together with astrocytes are capable of desaturation and elongation (Moore et al. 1991). 
For the synthesis of $20: 4 n-6, \Delta 6$-desaturase is the rate-limiting enzyme. It has gradual preferences for C18 fatty acids in the order $18: 3 n-3>18: 2 n-6>18: 1 n-9$ (Jeffcoat \& James, 1984). In addition, $n-3$ fatty acids inhibit the conversion of $18: 2 n-6$ to $18: 3 n-6$ (Garg et al. 1990), resulting in substrate accumulation and product reduction. This obviously led to the higher levels of $22: 5 n-3$ (results not shown) and $22: 6 n-3$ and reduction of $20: 4 n-6$ in all tissues examined in the present study.

It has been stated that the resistance to diet-induced changes of brain 22: $6 n-3$ develops after 3 weeks post-hatching (Anderson et al. 1992; Anderson, 1994). Our present results and an earlier report (Budowski et al. 1987) on the cerebellum with animals fed for 3 weeks confirm that the most prominent changes occur between weeks 2 and 3. However, in the liver the diet-induced changes occurred mainly during the first week. Notable was the intermediate effect of the oleic acid diet on $n-3$ and $n-6$ phospholipid fatty acids probably caused by the balanced $n-6: n-3$ value $(1 \cdot 2)$.

There is considerable evidence that vitamin $E$ influences tissue fatty acids (Infante, 1986). In liver and cerebrum increasing amounts of $\alpha$-tocopherol led to lower levels of 18:0, as recently described in the liver of rats (Clement \& Bourre, 1993). In the cerebellum $\alpha$ tocopherol had no effect. In liver $\alpha$-tocopherol affected $\Sigma n-3$ mainly after 1 week. An increasing effect of $\alpha$-tocopherol deficiency on $\Sigma n-3$ has been observed previously in rat liver and brain (Clement \& Bourre, 1993). It has been speculated (Witting, 1969) that during vitamin $\mathrm{E}$ deficiency the fatty acids are prone to peroxidative loss. The elevated levels observed are thought to be a reflection of an increased turnover of polyunsaturated fatty acids. Others (Buttriss \& Diplock, 1988) did not find a rise in $n-3$ fatty acids in $\alpha$ tocopherol-depleted rats.

According to our results 20:4n-6 was influenced by vitamin E, but only in liver. A rise of 20:4n-6 due to vitamin E deficiency has also been observed by others (Witting, 1969; Leat, 1983; Cunnane, 1988). This effect has been ascribed to the lack of inhibition of desaturases by $\alpha$-tocopherol. Others reported no alterations in rats (D'Aquino et al. 1985; Buttriss \& Diplock, 1988; Clement \& Bourre, 1993) and chicken (Klaus et al. 1995) or even a decrease that has been attributed to the lack of protection of the polyunsaturated fatty acids (D'Aquino et al. 1985; Buttriss \& Diplock, 1988). A reduction of $\alpha$-tocopherol produced lower levels of $\Sigma n-9$. This effect of $\alpha$-tocopherol on $n-9$ phospholipid fatty acids was found only in liver and cerebrum. Differences between tissues have been described previously (Clement \& Bourre, 1993). The probable underlying mechanism is the influence of $\Delta 9$-desaturase activity by $\alpha$-tocopherol (Okayasu et al. 1977).

Under the experimental conditions described here NE began at day 9. In previous experiments (Sallmann et al. 1991; Fuhrmann et al. 1994) with the same type of linoleic acid-rich fat but commercial chicks the disease started after $16 \mathrm{~d}$. So the onset of the disease obviously depends rather on the $\alpha$-tocopherol supply of the newly hatched chick than on the alterations of polyunsaturated fatty acids in the cerebellum as stated by Budowski et al. (1987).

According to our present results dietary fatty acids did modify tissue phospholipids extensively during the first 3 weeks of life. In liver the changes were most pronounced during the first week, whereas in brain the response was delayed. The UI of the cerebellum increased when both linolenic and linoleic acid were fed. Thus, the occurrence of NE in both deficient groups given fats rich in either 18:2 or 18:3 should be expected, because there is no difference in the tissue $\alpha$-tocopherol status due to the type of dietary fat (Fuhrmann \& Sallmann, 1995).

As an explanation for the occurrence of $\mathrm{NE}$ only with dietary linoleic, not linolenic acid, Budowski \& Crawford (1985) postulated a protective effect of $n-3$ fatty acids. Budowski et al. (1987) later stated that the lack of $\alpha$-tocopherol in the cerebellum leads to an increased 
peroxide tone. In a self-propagating process overproduction of eicosanoids derived from $20: 4 n-6$ should result in thrombus formation and ischaemia. The protective effect of $n-3$ fatty acids consists of inhibition of 20:4n-6 synthesis and promotion of the production of 20:5n-3 eicosanoids. However, according to the present results NE-producing dietary conditions were not accompanied by specific alterations in cerebellar phospholipid fatty acids in response to the $\alpha$-tocopherol content of the diet. The effect of $\alpha$-tocopherol on brain fatty acids seems to be limited to the saturated and monounsaturated fatty acids. However, this is not so in liver, where deficiency caused an increase of membrane unsaturation attributable to the pattern of $\Sigma n-3$ and $20: 4 n-6$. The results lead us to the assumption that $n-6$ fatty acids have a pro-oxidative-toxic effect stemming from $20: 4 n-6$ of the liver or arising in the cerebellum with its low content of $\alpha$-tocopherol. Previously Budowski et al. (1979) found that oxidation products from $n-6$ fatty acids were encephalopathogenic in vitamin E-deficient chicks. Therefore, the question of why only linoleic acid-rich fats induce the disease should concentrate now on toxic degradation products of $n-6$ fatty acids.

This work was supported by a grant from the Deutsche Forschungsgemeinschaft. The authors acknowledge the skilful technical assistance of Mr U. Glockenthoer. The reesterified soyabean triacylglycerol was generously supplied by Henkel Corp., Duesseldorf. All-rac- $\alpha$-tocopheryl acetate and Rovimix 428 without vitamin $E$ were a generous gift from Hoffmann-La Roche, Basle, Switzerland. The authors wish to thank Lohmann, Cuxhaven, for providing the breeder flock.

\section{REFERENCES}

Anderson, G. J. (1994). Developmental sensitivity of the brain to dietary $\mathrm{n} 3$ fatty acids. Journal of Lipid Research $35,105-111$.

Anderson, G. J., Connor, W. E. \& Corliss, J. D. (1990). Docosahexaenoic acid is the preferred dietary $n-3$ fatty acid for the development of the brain and retina. Pediatric Research 27, 89-97.

Anderson, G. J., Connor, W. E., Corliss, J. D. \& Lin, D. S. (1989). Rapid modulation of the $n-3$ docosahexaenoic acid levels in the brain and retina of the newly hatched chick. Journal of Lipid Research 30, 433-441.

Anderson, G. J., van Winkle, S. \& Connor, W. E. (1992). Reversibility of the effects of dietary fish oil on the fatty acid composition of the brain and retina of growing chicks. Biochimica et Biophysica Acta 1126, 237-246.

Aust, S. D., Chignell, C. F., Bray, T. M., Kalyanaraman, B. \& Mason, R. P. (1993). Free radicals in toxicology. Toxicology and Applied Pharmacology 120, 168-178.

Bartov, I. \& Bornstein, S. (1980). Susceptibility of chicks to nutritional encephalopathy: effect of fat and $\alpha$ tocopherol content of the breeder diet. Poultry Science 59, 264-267.

Bligh, E. G. \& Dyer, W. G. (1959). A rapid method of total lipid extraction and purification. Canadian Journal of Biochemistry and Physiology 37, 911-917.

Bourre, J.-M. \& Clement, M. (1991). Kinetics of rat peripheral nerve, forebrain and cerebellum $\alpha$-tocopherol depletion: comparison with different organs. Journal of Nutrition 121, 1204-1207.

Budowski, P., Bartov, I., Dror, Y. \& Frankel, E. N. (1979). Lipid oxidation products and chick nutritional encephalopathy. Lipids 14, 768-772.

Budowski, P. \& Crawford, M. A. (1985). $\alpha$-Linolenic acid as a regulator of the metabolism of arachidonic acid: dietary implications of the ratio, n-6:n-3 fatty acids. Proceedings of the Nutrition Society 44, 221-229.

Budowski, P., Leighfield, M. J. \& Crawford, M. A. (1987). Nutritional encephalomalacia in the chick: an exposure of the vulnerable period for cerebellar development and the possible need for both $\omega 6$ - and $\omega 3$-fatty acids. British Journal of Nutrition 58, 511-520.

Buttriss, J. L. \& Diplock, A. T. (1988). The relationship between $\alpha$-tocopherol and phospholipid fatty acids in rat liver subcellular membrane fractions. Biochimica et Biophysica Acta 962, 81-90.

Chamblee, T. N., Brake, J. D., Schultz, C. D. \& Thaxton, J. P. (1992). Yolk sac absorption and initiation of growth in broilers. Poultry Science 71, 1811-1816.

Clement, M. \& Bourre, J. M. (1993). Alteration of brain and liver microsomal polyunsaturated fatty acids following vitamin E deficiency. Neuroscience Letters 164, 163-166.

Cunnane, S. C. (1988). Vitamin E intake affects serum thromboxane and tissue essential fatty acid composition in the rat. Annals of Nutrition and Metabolism 32, 90-96.

D'Aquino, M., di Felice, M. \& Tomassi, G. (1985). Vitamin E status and effects of thermooxidised fats on structural $\alpha$-tocopherol and fatty acid of different rat tissues. Nutrition Reports International 32, $1179-1186$. 
DiMascio, P., Murphy, M. E. \& Sies, H. (1991). Antioxidant defense systems: the role of carotenoids, tocopherols, and thiols. American Journal of Clinical Nutrition 53, S194-S200.

Friedman, P. (1986). Dynastat Professional Statistics and Graphics, version 3.3. Washington, DC: Dynamic Microsystems Inc.

Fuhrmann, H., Balthazary, S. T. \& Sallmann, H. P. (1994). Bioefficiency of different tocopherols as assessed by haemolysis test and microsomal pentane production. British Journal of Nutrition 71, 605-614.

Fuhrmann, H. \& Sallmann, H. P. (1995). $\alpha$-Tocopherol and phospholipase $A_{2}$ in liver and brain of chicks posthatching: the influence of dietary fat and vitamin E. Annals of Nutrition and Metabolism 39, 302-309.

Garg, M. L., Thomson, A. B. R. \& Clandinin, M. T. (1990). Interactions of saturated, $n-3$ and $n-6$ polyunsaturated fatty acids to modulate arachidonic acid metabolism. Journal of Lipid Research 31, 271-277.

Hassan, S., Hakkarainen, J., Jönsson, L. \& Työppönen, J. (1990). Histopathological and biochemical changes associated with selenium and vitamin E deficiency in chicks. Journal of Veterinary Medicine A 37, 708-720.

Infante, J. P. (1986). Vitamin E and selenium participation in fatty acid desaturation. A proposal for an enzymatic function of these nutrients. Molecular and Cellular Biochemistry 69, 93-108.

Jeffcoat, R. \& James, A. T. (1984). The regulation of desaturation and elongation of fatty acids in mammals. In Fatty Acid Metabolism and its Regulation, pp. 85-112 [S. Numa, editor]. Amsterdam: Elsevier Science Publishers.

Kaluzny, M. A., Duncan, L. A., Merrit, M. V. \& Epps, D. E. (1985). Rapid separation of lipid classes in high yield and purity using bonded phase columns. Journal of Lipid Research 26, 135-144.

Klaus, A.-M., Fuhrmann, H. \& Sallmann, H. P. (1995). Peroxidative and antioxidative metabolism of the broiler chicken as influenced by dietary linoleic acid and vitamin E. Archiv fur Gefluegelkunde 59, 135-144.

Leat, W. M. F. (1983). Nutritional deficiencies and fatty acid metabolism. Proceedings of the Nutrition Society 42 , 333-342.

Lepage, G. \& Roy, C. C. (1986). Direct transesterification of all classes of lipids in a one-step reaction. Journal of Lipid Research 27, 114-120.

Lin, D. S., Connor, W. E. \& Anderson, G. J. (1991). The incorporation of $n-3$ and $n-6$ essential fatty acids into the chick embryo from egg yolks having vastly different fatty acid compositions. Pediatric Research 29, 601-605.

Lowry, O. H., Rosebrough, N. J., Farr, A. L. \& Randall, R. J. (1951). Protein measurement with the Folin phenol reagent. Journal of Biological Chemistry 193, 265-275.

Moore, S. A., Yoder, E., Murphy, S., Dutton, G. R. \& Spector, A. A. (1991). Astrocytes, not neurons, produce docosahexaenoic acid $(22: 6 \omega-3)$ and arachidonic acid $(20: 4 \omega-6)$. Journal of Neurochemistry 56, 518-524.

Noble, R. C., Cocchi, M. \& Bath, H.M. (1993). $\alpha$-Tocopherol absorption and polyunsaturated fatty acid metabolism in the developing chick embryo. British Poultry Science 34, 815-818.

Okayasu, T., Kameda, K., Ono, T. \& Imai, Y. (1977). Effect of dietary vitamin B2 and vitamin E on the delta9-desaturase and catalase activities in rat liver microsomes. Biochimica et Biophysica Acta 489, 379-402.

Sallmann, H. P., Fuhrmann, H., Molnar, S. \& Stegmanns, T. (1991). Endogenous lipid peroxidation in broiler chickens under dietary loads. Fat Science and Technology 93, 457-462.

Sardesai, V. M. (1992). Nutritional role of polyunsaturated fatty acids. Journal of Nutritional Biochemistry 3, $154-166$.

Witting, L. A. (1969). Effect of antioxidant-deficiency on phospholipid composition in the chick brain. Journal of Neurochemistry 16, 1253-1256. 